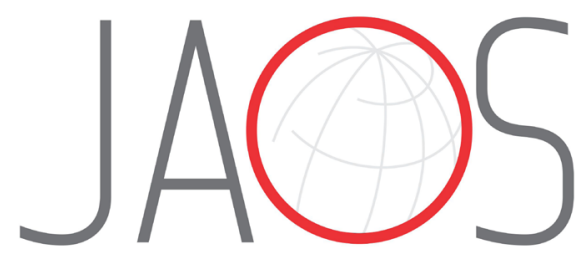

JOURNAL OF APPLIED ORAL SCIENCE

\title{
Physicochemical properties and biological effects of quaternary ammonium methacrylates in an experimental adhesive resin for bonding orthodontic brackets
}

\section{Abstract}

Tatiana Féres ASSAD-LOSS ${ }^{1}$

Jamille Ferreira VIGNOLI ${ }^{1}$

Isadora Martini GARCIA²

Maristela Barbosa PORTELA ${ }^{1}$

Luis Felipe J. SCHNEIDER ${ }^{1,3}$

Fabrício Mezzomo COLLARES ${ }^{4}$

Larissa Maria Assad CAVALCANTE ${ }^{1,3}$

Monica de Almeida TOSTES 4 iD

Submitted: December 10, 2020

Modification: January 27, 2021

Accepted: February 10, 2021

Corresponding address: Tatiana Féres Assad-Loss Av. Jornalista Alberto Francisco Torres, 469/602 - 24230-008 Icaraí - Niterói - RJ - Brasil. email: tatianaassad@yahoo.com.br
Fixed orthodontic appliances may lead to biofilm accumulation around them that may increase caries risk. This study aimed to evaluate the influence of quaternary ammonium methacrylates (QAMs) on the physicochemical properties, cytotoxicity, and antibacterial activity of adhesive resins for orthodontic purposes. Methodology: A base resin was prepared with a comonomer blend and photoinitiator/co-initiator system. Two different QAMs were added to the base adhesive: dimethylaminododecyl methacrylate at 5 wt.\% (DMADDM) or dimethylaminohexadecyl methacrylate (DMAHDM) at 10 wt.\%. The base adhesive, without QAMs, (GC) and the commercial Transbond $^{\mathrm{TM}} \mathrm{XT}$ Primer $3 \mathrm{M}$ (GT) were used as control. The resins were tested immediately and after six months of aging in the water regarding the antibacterial activity and shear bond strength (SBS). The antibacterial activity was tested against Streptococcus mutans via metabolic activity assay (MTT test). The groups were also tested for the degree of conversion (DC) and cytotoxicity against keratinocytes. Results: The resins containing QAM showed antibacterial activity compared to the commercial material by immediately reducing the metabolic activity by about $60 \%$. However, the antibacterial activity decreased after aging $(p<0.05)$. None of the groups presented any differences for SBS $(p>0.05)$ and DC $(p>0.05)$. The incorporation of DMADDM and DMAHDM significantly reduced the keratinocyte viability compared to the GT and GC groups $(p<0.05)$. Conclusion: Both adhesives with QAMs showed a significant reduction in bacterial metabolic activity, but this effect decreased after water aging. Lower cell viability was observed for the group with the longer alkyl chain-QAM, without significant differences for the bonding ability and degree of conversion. The addition of QAMs in adhesives may affect the keratinocytes viability, and the aging effects maybe decrease the bacterial activity of QAM-doped materials.

Keywords: Orthodontics. Dental Bonding. Dental caries. Quaternary Ammonium Compounds. Light-Curing of Dental Adhesives.

${ }^{1}$ Universidade Federal Fluminense, Programa de pós-graduação em Odontologia, Niterói, RJ, Brasil. 2Universidade Federal do Rio Grande do Sul, Laboratório de Materiais Dentários, Porto Alegre, RS, Brasil.

3Universidade Veiga de Almeida, Pós-graduação em Odontologia, Rio de Janeiro, RJ, Brasil.

${ }^{4}$ Universidade Federal do Rio Grande, Pós-Graduação em Odontologia da do Sul, Porto Alegre, RS, Brasil. 


\section{Introduction}

Fixed orthodontic treatment leads to significant caries risk due to biofilm accumulation around the appliance components and the challenges to achieve a reliable oral hygiene. ${ }^{1-5}$ During orthodontic bonding procedures, the excess of adhesive is invariably left on the tooth surface at the bracket-enamel interface. This adhesive excess may be a site for rapid attachment and growth of oral microorganisms such as Streptococcus mutans. ${ }^{6}$ Demineralization occurs when the oral environment $\mathrm{pH}$ favors calcium and phosphate ions out of enamel, and early lesions appear clinically as opaque white spots caused by mineral loss. ${ }^{1}$ White spot lesions (WSL) are one of the most common adverse effects of orthodontic treatment, 1,5 occurring in almost $50 \%$ of patients during the first year of treatment ${ }^{4}$ and may negatively affect dental esthetics. ${ }^{1}$ Unfortunately, preventive approaches during orthodontic treatments have not been effective, and the occurrence of WSL is positively and significantly associated with the duration of the therapy. ${ }^{1,7}$

Antibacterial materials could be a promising alternative to biofilm control in orthodontics patients. ${ }^{8,9}$ The effectiveness of different antibacterial materials added to adhesives has been evaluated in vitro, $8,10-$ 13 in situ, ${ }^{10}$ and in vivo, ${ }^{9}$ showing positive results to decrease biofilm viability. However, the duration of the antibacterial effect of resins remains uncertain. Among the antibacterial agents that have been tested, the incorporation of methacrylate monomers derived from quaternary ammonium salts has increasing attention. Quaternary ammonium methacrylates (QAM) present antimicrobial mechanisms by disturbing the electrical balance of bacterial membrane through their positively charged molecules with the negatively charged compounds on the bacteria surface. This event leads to bacterial membrane disruption and cell death. ${ }^{13-16}$ Besides providing antibacterial activity, the incorporation of antimicrobial agents should not alter dental material physical properties. ${ }^{15,16}$ For orthodontic purposes, the shear bond strength (SBS) between the adhesive and the bracket should not be affected.

However, the literature is divergent regarding the most appropriate QAM and its concentration to be added in adhesive resins for bonding orthodontic brackets. ${ }^{11,12,17}$ Previously, the physical properties and antimicrobial activity of two QAM dimethylaminododecyl methacrylate (DMADDM) and dimethylaminohexadecyl methacrylate (DMAHDM) were tested at two different concentrations (5 wt.\% or 10 wt.\%). ${ }^{18,19}$ The longer the carbon chain of the QAM and the higher its concentration, the higher the antimicrobial capacity achieved. However, the physical properties of the resin materials were jeopardized by increasing QAM concentration. DMADDM at $5 \mathrm{wt} \%$, in turn, led to the lowest polymer degradation after water storage among groups ${ }^{19}$ while maintaining the antimicrobial property. ${ }^{18}$ However, there is no evaluation of SBS nor cytotoxicity of these adhesives. Our study aimed at evaluating the influence of quaternary ammonium methacrylates (QAM) in the physicochemical properties, cytotoxicity, and antibacterial activity of adhesive resins for orthodontic purposes. For this purpose, we evaluated the addition of DMADDM at 5 wt. $\%$ or DMAHDM at 10 wt. $\%$ in the base resin considering both the immediate and the long-term antibacterial property, along with SBS. We also analyzed the degree of conversion and cytotoxicity against human keratinocytes. The null hypothesis is that QAM addition in the experimental adhesive will not affect physicochemical properties tested and will not promote antibacterial activity and cytotoxic effect.

\section{Methodology}

\section{Synthesis of antibacterial monomers}

Menschutkin reaction was used to synthesize the antimicrobial monomers, as reported 14,20 and previously published. ${ }^{18,19}$ A tertiary amine and an organo-halide were added to this reaction, in equal amounts $(60 \mathrm{mmol})$, into a round bottom flask coupled to a condenser with $20 \mathrm{~mL}$ of ethanol and were refluxed for $24 \mathrm{~h}$. The pure monomer was obtained when the solvent was evaporated in a rotatory evaporator until dryness and so it did not need purification. For each monomer, a different organo-halide was used, since the tertiary amine was always 2-(dimethylamino)ethyl methacrylate (DMAEMA).

\section{Experimental adhesives formulation}

The adhesive formulation 21,22 was the mixture of bisphenol-A-glycidyldimetacrylate (Bis-GMA, Esstech, Essington, Pennsylvania, USA) and 2-hydroxyethylmethacrylate (HEMA, Esstech, Essington, Pennsylvania, USA) at a 55:45 wt.\% ratio. To this base resin, $0.5 \mathrm{~mol} \%$ camphorquinone 
(Esstech Inc., Essington, Pennsylvania, USA) and 1 mol\% EDMAB amine (Sigma-Aldrich, St Louis, Missouri, USA) were added as the photoinitiator/coinitiator system. The synthesized QAMs were added at $5 \mathrm{wt}$ \% DMADDM or $10 \mathrm{wt} \%$ DMAHDM of the total organic matrix weight. The groups were arranged as shown in Figure 1:

\begin{tabular}{|c|c|}
\hline Groups & Composition \\
\hline GT & $\begin{array}{c}\text { Commercial adhesive Transbond XT Primer (3M } \\
\text { Unitek, Monrovia, California, USA) }\end{array}$ \\
\hline GC & Experimental adhesive without QAM \\
\hline G12C/5 & Experimental adhesive with 5 wt.\% of DMADDM \\
\hline G16C/10 & Experimental adhesive with 10 wt.\% of DMAHDM \\
\hline
\end{tabular}

Figure 1- Composition of each adhesive

\section{Antibacterial activity}

Forty-eight adhesive discs were prepared to evaluate the immediate (T0) and long-term (T1) antibacterial activity $(n=6)$. Metallic molds measuring $2 \mathrm{~mm}$ thickness and $1 \mathrm{~mm}$ diameter were used. The photoactivation was performed for $20 \mathrm{~s}$ on each side of the samples using a light-emitting diode (Emitter B Supra, Schuster Equipamentos Odontológicos Ltda, Santa Maria, RS, Brazil) with $1.200 \mathrm{~mW} / \mathrm{cm}^{2}$ (measured with a radiometer, RD-7, Ecel, Ribeirão Preto, SP, Brazil). This device was used throughout the whole study. The samples for long-term analysis were stored in distilled water at $37^{\circ} \mathrm{C}$ for six months. The discs were sterilized with UV light for 1 hour and placed in sterile flat-bottom 24-well plates. ${ }^{23}$ On each well with sterilized adhesive discs, $2 \mathrm{~mL}$ of bacterial suspension in Brain Heart Infusion broth (BHI, Difco, Sparks, Nevada, USA) supplements with 2 wt.\% sucrose were added. The strain of Streptococcus mutans ATCC 25175 (American Type Culture Collection, Fiocruz, Rio de Janeiro, RJ, Brazil) used was cultured in $\mathrm{BHI}$ at $37^{\circ} \mathrm{C}$ for $24 \mathrm{~h}$ under low oxygen tension conditions. The bacterial suspension was obtained by adjusting the bacterial growth to 0.5 following the McFarlane seal at $550 \mathrm{~nm}$ using UV/Vis spectrophotometer. Next, this suspension was diluted $1: 100$, and $10 \mu \mathrm{L}$ of the suspension was added to each well, containing an adhesive disc with $2 \mathrm{~mL}$ of $\mathrm{BHI}$ broth supplemented with $2 \mathrm{wt}$. \% sucrose. The 24-well plates were rapt for $48 \mathrm{~h} \mathrm{t} 37^{\circ} \mathrm{C}$ under microaerophilic conditions. Then, 3-[4,5-dimethylthiazol-2-yl]2,5-diphenyl tetrazolium bromide (MTT, $1 \mathrm{mg} / \mathrm{mL}$ in PBS) was added to each well and incubated at the same conditions for $1 \mathrm{~h}$. Subsequently, $1 \mathrm{~mL}$ of dimethylsulfoxide (DMSO) was added to each well, and the plates were incubated for $20 \mathrm{~min}$ at room temperature. The suspensions were subjected to a spectrophotometer at $540 \mathrm{~nm}$ (DU 530, Beckman Coulter Company, Brea, CA, USA). A higher absorbance was indicative of a higher concentration of formazan, which, in turn, indicated a higher metabolic activity of $S$. mutans biofilm. ${ }^{18,19}$

\section{Shear bond strength (SBS)}

Ninety-six bovine incisors were used to evaluate both the immediate (TO) and the long-term (T1) SBS $(n=12)$. The teeth were fixed with polystyrene resin using a PVC matrix of $20 \mathrm{~mm}$ in height and $25 \mathrm{~mm}$ in diameter with the vestibular face parallel to the resin base. The labial surfaces of the teeth were polished with water sandpaper of 400 and 600 grain to obtain a flat surface and make a uniform smear layer, rubber brush and pumice. Then, preconditioned with $37 \%$ phosphoric acid for a period of $30 \mathrm{~s}$, rinsed with water for $30 \mathrm{~s}$ and air-dried. Next, one layer of the adhesive, according to the groups, was applied and air-dried. Brackets (Edgewise, Morelli, Sorocaba, Brazil) were fixed to the centers of the teeth vestibular faces using the same resin cement Transbond XT (3M Unitek, Monrovia, California, USA) for all groups. The resin cement was photoactivated for $10 \mathrm{~s}$ on each face of the bracket; thus, the photoactivation time totaled 40 s. The samples were subjected to the SBS test in a universal test machine (EMIC, São José dos Pinhais, PR, Brazil). A knife-edge chisel $(0.1 \mathrm{~mm})$ applied at $180^{\circ}$ to the tooth labial face was positioned at the adhesive-enamel interface. The force was applied with $0.5 \mathrm{~mm} / \mathrm{min}$ until the moment the bracket was detached. The results were recorded in MPa.

\section{Degree of conversion (DC)}

The orthodontic adhesives $(n=3)$ were placed on the attenuated total reflectance device of a Fourier transformed infrared (FTIR) spectrometer (Alpha-P; Bruker Optics, Ettlingen, Germany). Each sample was photoactivated for $40 \mathrm{~s}$ using the light-emitting diode. The photoactivation was standardized at 1 $\mathrm{mm}$ between the light-unit tip and the samples. The same light-curing unit, irradiance, and radiometer were used for all subsequent methodologies. Spectra from FTIR analysis were recorded before and 1 minute after photoactivating each sample with 32 scans and a $4 \mathrm{~cm}^{-1}$ resolution. The DC was estimated based on the area of the $1638 \mathrm{~cm}^{-1}$ peak (carbon-carbon 
double bonds of aliphatic chain) and $1608 \mathrm{~cm}^{-1}$ peak (carbon-carbon double bonds of aromatic chain) using the following formula: was added $(50 \mu \mathrm{L})$ in each well, and the plates were incubated for $30 \mathrm{~min}$ at room temperature. The plates were washed with $1 \%$ acetic acid solution four times

$$
\mathrm{DC}(\%)=100 \times\left(\frac{\text { peak height of cured aliphatic } \mathrm{C}=\mathrm{C} / \text { peak height of cured aromatic } \mathrm{C}=\mathrm{C}}{\text { peak height of uncured aliphatic } \mathrm{C}=\mathrm{C} / \text { peak height of uncured aromatic } \mathrm{C}=\mathrm{C}}\right)
$$

\section{Cytotoxicity evaluation}

To evaluate a possible cytotoxic effect, the adhesives $(n=5)$ were prepared ( $2 \mathrm{~mm}$ thickness and $4 \mathrm{~mm}$ diameter) by photoactivation during 20 $\mathrm{s}$ on each side. The samples were stored for $24 \mathrm{~h}$ in distilled water at $37^{\circ} \mathrm{C}$ and sterilized with hydrogen peroxide plasma $(58 \%)$ for $48 \mathrm{~min}$ at $56^{\circ} \mathrm{C} .{ }^{24}$ The cytotoxicity evaluation was performed against human keratinocytes (HaCaT, CLS Cell Lines Service GmbH, Eppelheim, Baden-Württemberg, Germany). One hundred microliters of Dulbecco's Modified Eagle Medium (DMEM, Thermo Scientific, Waltham, MA, USA) with HaCat were inoculated in 96-well plates at $5 \times 10^{3}$ cells/per well. The plates were kept at $37^{\circ} \mathrm{C}$ for $24 \mathrm{~h}$. The samples were individually immersed in Eppendorf tubes with $1 \mathrm{~mL}$ of DMEM and kept at $37^{\circ} \mathrm{C}$ for $24 \mathrm{~h}$ to prepare the eluates. Then, $100 \mu \mathrm{L}$ from each Eppendorf tube was placed in contact with HaCat in the wells. Five aliquots of $100 \mu \mathrm{L}$ were tested from each sample, totalizing twenty-five wells per group. One group was used as control: wells with HaCat and DMEM without eluates. The plates were kept at $37^{\circ} \mathrm{C}$ for $72 \mathrm{~h}$. After this period, the cells were fixed with $50 \mu \mathrm{L}$ of trichloroacetic acid (TCA) solution (1 TCA: 1 distilled water) and kept at $4^{\circ} \mathrm{C}$ for one hour. The plates were washed with running water six times and left at room temperature to dry. Sulforhodamine B (SRB) solution at $0.4 \%$ (diluted in acetic acid solution at $1 \%$ ) and dried at room temperature. Trizma solution $10 \mathrm{mM}$ $(100 \mu \mathrm{L})$ was added and the plates were incubated for $1 \mathrm{~h}$ at room temperature. The plates were analyzed at $560 \mathrm{~nm}$ to obtain the absorbance value from each well. The cell viability was normalized against the viability of cells in wells without eluates (control of the test) and expressed in percentage of viability. ${ }^{24}$

\section{Statistical Analysis}

The data were analyzed using the software SigmaPlot, version 12.0 (Systat Software, San Jose, CA, USA). Data distribution was evaluated using the Shapiro-Wilk test. Data from the antibacterial activity and SBS were analyzed using two-way ANOVA and Tukey's test for post-hoc analysis considering the two factors: "time" and "orthodontic adhesive". The DC and the cytotoxicity were analyzed using one-way ANOVA and Tukey's test. All analyses were performed at a 5 $\%$ significance level.

\section{Results}

Figure 2 shows the results of antibacterial activity for the orthodontic adhesive resins. The interaction between the variables "time" and "orthodontic adhesive" indicated a statistically significant level at

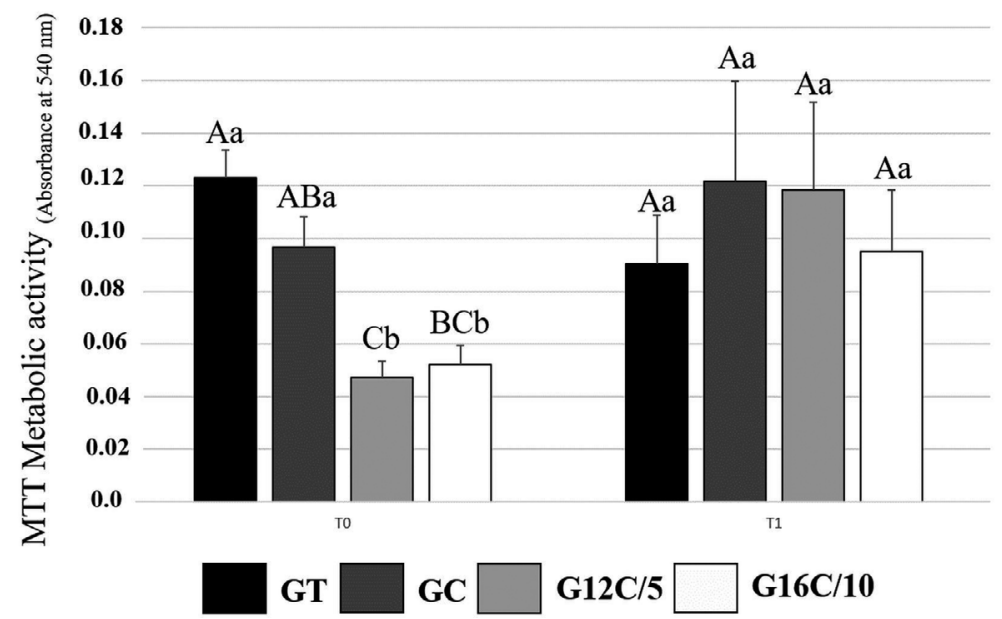

Figure 2- Results of antibacterial activity of the orthodontic adhesive resins. The values of optical density immediately (T0) and after six months of aging in distilled water (T1) are expressed in mean and standard deviation of absorbance values. Different capital letters indicate statistically significant differences among the materials within the same time $(p<0.05)$. Different small letters indicate a statistically significant difference for the same adhesive, comparing the results of different times $(p<0.05)$ 


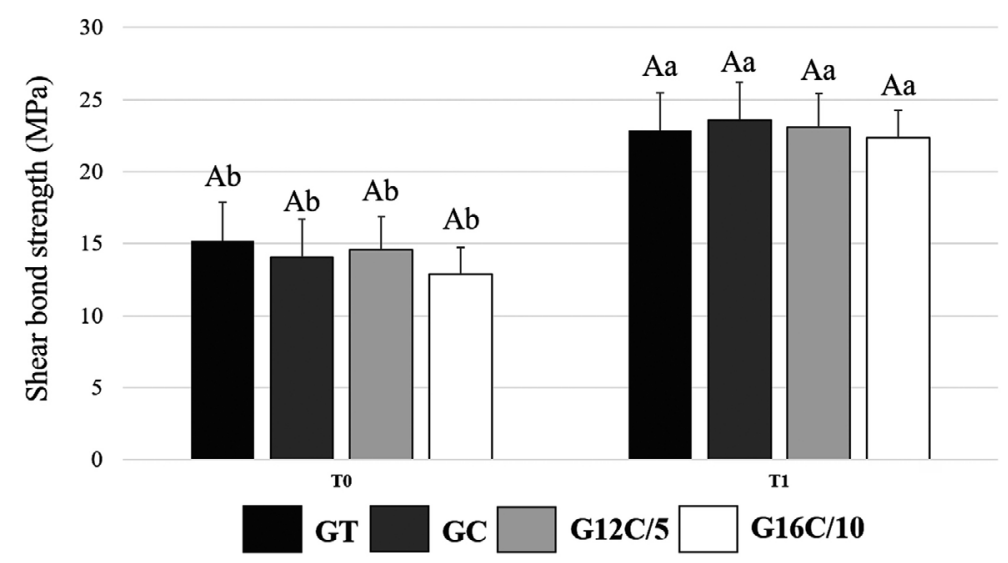

Figure 3- Results of shear bond strength (SBS) immediately (T0) and after six months (T1) of aging in distilled water. The values of SBS are expressed in mean and standard deviation of MPa. Same capital letters indicate no statistically significant differences among the materials within the same time $(p>0.05)$. Different small letters indicate statistically significant difference for the same adhesive comparing the results of different times $(p<0.05)$

Table 1- Mean and standard deviation values of degree of conversion (DC) and cell viability of the orthodontic adhesive resins

\begin{tabular}{ccc}
\hline Groups & DC (\%) & Cell Viability (\%) \\
\hline GT & $73.9( \pm 0.7)^{\mathrm{A}}$ & $102.1( \pm 5.0)^{\mathrm{A}}$ \\
GC & $76.2( \pm 0.8)^{\mathrm{A}}$ & $79.4( \pm 5.1)^{\mathrm{B}}$ \\
$\mathrm{G} 12 \mathrm{C} / 5$ & $72.2( \pm 1.7)^{\mathrm{A}}$ & $43.0( \pm 9.4)^{\mathrm{C}}$ \\
$\mathrm{G} 16 \mathrm{C} / 10$ & $78.6( \pm 0.5)^{\mathrm{A}}$ & $12.5( \pm 3.4)^{\mathrm{D}}$ \\
\hline
\end{tabular}

$p<0.05$. The effect of different levels of "orthodontic adhesive" depended on what level "time" was present. The groups containing DMAHDM or DMADDM showed around $60 \%$ lower metabolic activity compared to GT in T0, with a statistically significant difference between DMADDM and GT to GC ( $<<0.05)$, without statistical difference between DMADDM and DMAHDM ( $p>0.05)$. After six months of aging in distilled water (T1), the biofilms formed on the samples with DMAHDM or DMADDM showed higher metabolic activity compared to the immediate analysis (T0) $(p<0.05)$. In T1, there was no statistically significant difference among groups $(p>0.05)$.

Figure 3 shows the results of SBS immediately (TO) and after six months (T1) of aging in distilled water. In T0, the values ranged from 12.9 ( \pm 1.9 ) MPa for $\mathrm{G} 16 \mathrm{C} / 10$ to 15.2 ( \pm 2.7 ) MPa for GT. In T1, the values ranged from $22.3( \pm 2.7) \mathrm{MPa}$ for $\mathrm{G} 16 \mathrm{C} / 10$ to $23.6( \pm 4.3) \mathrm{MPa}$ for GC. There was no statistical difference among groups in T0 ( $p>0.05)$. Moreover, no differences were found among groups in T1 ( $p>0.05)$. All groups had an increased value of SBS between T0 and T1 $(p<0.05)$.

Table 1 shows the results of DC of the orthodontic adhesive resins. The values ranged from $72.2( \pm 1.7)$ $\%$ for $\mathrm{G} 12 \mathrm{C} / 5$ to 78.6 ( \pm 0.5$) \%$ for $\mathrm{G} 16 \mathrm{C} / 10$. There was no statistical difference among groups for the DC $(p>0.05)$. Table 1 also shows the results of cell viability. The values ranged from 102.1 ( \pm 5.0$) \%$ for GT to 12.5 $( \pm 3.4) \%$ for $\mathrm{G} 16 \mathrm{C} / 10$, with statistically significant differences among groups ( $p<0.05)$. G16C/10 showed the lowest value among the adhesives, which was statistically lower than G12C/5 (43.0 $9.4 \%)$.

Same letters indicate no statistical difference among groups in the same column ( $p>0.05)$.

Different letters indicate statistical difference among groups in the same column $(p<0.05)$.

The cell viability was normalized against the viability of cells in wells without eluates (control of the test) and expressed in percentage of viability.

\section{Discussion}

WSL around brackets are a common complication of treatment with fixed orthodontic appliances. ${ }^{4,7}$ Due to its sealing capacity and antibacterial activity, the application of an adhesive containing QAM may be a promising strategy for the management of WSL. This approach may assist in preventing caries lesions in non-cooperative patients. ${ }^{11,12,25}$ In our study, the addition of QAM did not change the physicochemical properties evaluated, and antibacterial activity was achieved in comparison to the commercial orthodontic adhesive. However, cytotoxic effect against human keratinocytes and decreased antibacterial activity were observed after six months of water aging.

An excess adhesive flash promotes a rough site susceptible to the attachment and growth of $S$. mutans. ${ }^{6}$ However, antibacterial polymerized adhesives 
could inhibit bacteria attachment on enamel margins of the bracket, ${ }^{13}$ which may be especially useful for the site with an excess of adhesive. DMAHDM is a QAM with an alkyl chain length composed of sixteen carbons. Previous studies showed that this QAM exhibits a higher antibacterial effect comparison to DMADDM. ${ }^{13,15}$ There were no significant differences when the group of 5 wt. \% of DMADDM was compared with 10 wt.\% of DMAHDM, which differs from our previous study. ${ }^{18}$ Although there was no statistically significant difference between $\mathrm{G} 16 \mathrm{C} / 10$ and the control groups in T0, the percentage difference was high (around $60 \%$ compared to GT), and there was no statistical difference among both adhesives containing QAM. In our study, we used MTT assay to evaluate the antibacterial activity. In this test, the reduction of tetrazolium salts to formazan is associated with microorganism metabolic activity. A higher absorbance in the MTT assay suggests a higher formazan concentration and higher metabolic activity. However, this test does not measure cell viability, such as colonyforming units assay. As an initial test to compare the antibacterial activity immediately and over time, it is interesting to use MTT because the pathogenicity of S. mutans is related to its metabolic activity and acid production. ${ }^{26}$ Further studies to evaluate the developed materials via colony-forming unit tests would assist in understanding the antibacterial effects. ${ }^{25}$

After water aging, the effect was lower, and no difference was observed among groups. According to Rego, et al. ${ }^{18}$ (2017) the composites containing QAM showed antibacterial activity after brushing simulation. However, there was an increase in viable biofilm after brushing and polishing, suggesting that the antibacterial effects of composites with QAM decrease over time. ${ }^{18}$ The effect of water in the chemical and structure of the polymer network is essential to understand the possible behavior of dental materials in the oral environment. ${ }^{27}$ Besides the aging performed in our study, the effect of degradation was previously demonstrated to reduce the surface hardness, increase sorption and solubility, and hygroscopic expansion. ${ }^{19}$ The leaching part of the QAM from the cured adhesive surface may explain the weakened effect of antibacterial activity after water-aging in our study. ${ }^{13}$ Even with methacrylate groups, part of the QAM may not have copolymerized the resin and was lost during the storage.

There is no standard method for assessing the SBS of brackets to enamel since the loads applied in clinical settings on brackets are dynamic and complex. ${ }^{28}$ A mixture of shear and tensile loads are applied to brackets in vivo, and they cannot be perfectly simulated in vitro. The forces during chewing are abrupt and impose a high risk of damage to the enamel when compared with the force applied during the SBS test. ${ }^{28,29}$ However, this is the most widely used assay to assess bonding effectivenesss of orthodontics. ${ }^{8}$ In our study, the SBS results showed no differences among groups, indicating that the addition of the QAM did not affect the mechanical property of the material, corroborating with previous studies. ${ }^{28,30,31}$

In the immediate SBS, there was no difference among groups. Interestingly, all adhesives showed higher SBS outcomes after six months. The rationale for that may be delayed in the polymerization reaction when the specimens were stored in warm distilled water over time. ${ }^{32}$ The addition of DMADDM at $5 \mathrm{wt} . \%$ also resulted in less degradation for the composite in a previous study. ${ }^{19}$ Besides the non-difference observed for the SBS among groups in the immediate or in the long-term analysis, the DC of the orthodontic resins also did not change with the addition of QAM. This property is related to the chemical and mechanical properties of the polymers, such as diametric tensile strength, compressive strength, hardness, flexural modulus, and strength. ${ }^{27}$ In our study, all groups showed DC similar to commercial ${ }^{33}$ and experimental ${ }^{34}$ adhesives reported in the literature. The higher DC (up to $70 \%$ ) observed for all groups may have assisted in the achievement of reliable SBS over time since the higher the DC, the higher the mechanical properties and hydrolytic stability of adhesives. ${ }^{34}$

Cytotoxicity of the adhesives against keratinocytes was evaluated because these cells are present in the oral mucosa. Viable cells were identified using the SRB dye test. Compared to the MTT assay, which is another colorimetric test and an ISO-indicated method. The SRB test has better predictive power by marking viable cell proteins and not relying on cell metabolism for MTT assay. In our study, the cell viability was lower than ISO requires (inferior to $70 \%$ is considered a cytotoxic material when the MTT test is applied), indicating that, even with copolymerizable QAM, probably some of these components may leach from the resin matrix. However, unlike most studies, we used pure eluates on the cells for $72 \mathrm{~h}$ without further dilution. Moreover, the samples used had a large area in contact with DMEM 
for $24 \mathrm{~h}$ to produce the eluate, increasing the chances of forming a DMEM with more leached compounds from the specimens and increasing cytotoxic effects. Quantifying the leached compounds of QAMs from the resins was beyond the scope of this study; however, future investigations are encouraged to analyze the leached compounds over time and their correlation with antibacterial and cytotoxic activity.

Even though we evaluated the cytotoxic potential of the adhesives with the highest possible challenge against the cells, careful application on tooth surfaces is recommended. However, the amount of adhesive used in a bracket bonding is only one thin layer in contrast to $4 \mathrm{~mm} \times 2 \mathrm{~mm}$ of adhesives discs tested in the study. Other authors tested QAM against fibroblast and odontoblasts, and there were no differences in cytotoxicity compared to commercial adhesives. ${ }^{15}$ On the other hand, our experimental adhesive without QAM ${ }^{21,22}$ also showed a decrease in cell viability, suggesting that other experimental adhesive components could be involved in this process and lead to cytotoxicity.

\section{Conclusions}

In our study, the effects of two different QAMs in an adhesive for orthodontic purposes were evaluated. DMADDM at 5 wt. $\%$ and DMAHDM at 10 wt. \% were incorporated into an adhesive blend. The physicochemical and antibacterial properties, besides the cytotoxicity, were analyzed. In short, both adhesives with QAMs showed a significant reduction in bacterial metabolic activity, but this effect decreased after water aging. Lower cell viability was observed for the group with the longer alkyl chain-QAM, without significant differences for the bonding ability and degree of conversion. The addition of QAMs in adhesives may affect the keratinocytes viability, and the aging effects may decrease the bacterial activity of QAMs-doped materials.

\section{Authors' contributions}

Assad-Loss, Tatiana: Conceptualization (Equal); Data curation (Equal); Formal analysis (Equal); Funding acquisition (Equal); Investigation (Equal); Methodology (Equal); Resources (Equal); Writingoriginal draft (Equal). Ferreira, Jamille Vignoli: Data curation (Equal); Methodology (Equal);
Writing-original draft (Equal); Garcia, Isadora Martini: Conceptualization (Equal); Data curation (Equal);Formal analysis (Equal); Investigation (Equal); Methodology (Equal); Resources (Equal); Software (Equal); Writing-original draft (Equal); Portela, Maristela Barbosa: Conceptualization (Equal); Data curation (Equal); Formal analysis (Equal); Investigation (Equal); Methodology (Equal); Resources (Equal); Schneider, Luis Felipe: Conceptualization (Equal); Data curation (Equal); Formal analysis (Equal); Investigation (Equal); Methodology (Equal); Resources (Equal); Software (Equal); Validation (Equal); Writing-review \& editing (Equal). Collares, Fabrício M.: Conceptualization (Equal); Project administration (Equal); Supervision (Equal); Validation (Equal); Visualization (Equal); Writing-review \& editing (Equal); Cavalcante, Larissa: Conceptualization (Equal); Formal analysis (Equal); Investigation (Equal); Project administration (Equal); Supervision (Equal); Validation (Equal); Visualization (Equal); Writing-review \& editing (Equal); Tostes, Monica Almeida: Conceptualization (Equal); Formal analysis (Equal); Investigation (Equal); Methodology (Equal); Project administration (Equal); Supervision (Equal); Validation (Equal); Visualization (Equal); Writingreview \& editing (Equal).

\section{References}

1- Heymann GC, Grauer D. A contemporary review of white spot lesions in orthodontics. J Esthet Restor Dent. 2013;25(2):85-95. doi: $10.1111 /$ jerd. 12013

2- Julien KC, Buschang PH, Campbell PM. Prevalence of white spot lesion formation during orthodontic treatment. Angle Orthod. 2013;83(4):641-7. doi: 10.2319/071712-584.1

3- Maxfield BJ, Hamdan AM, Tufekci E, Shroff B, Best AM, Lindauer SJ. Development of white spot lesions during orthodontic treatment: perceptions of patients, parents, orthodontists, and general dentists. Am J Orthod Dentofacial Orthop. 2012;141(3):337-44. doi: 10.1016/j. ajodo.2011.08.024

4- Tufekci E, Dixon JS, Gunsolley JC, Lindauer SJ. Prevalence of white spot lesions during orthodontic treatment with fixed appliances. Angle Orthod. 2011;81(2):206-10. doi: 10.2319/051710-262.1

5- Altmann AS, Collares FM, Leitune VC, Arthur RA, Takimi AS, Samuel SM. In vitro antibacterial and remineralizing effect of adhesive containing triazine and niobium pentoxide phosphate inverted glass. Clin Oral Investig. 2017;21(1):93-103. doi: 10.1007/s00784-0161754-y

6- Ho CS, Ming Y, Foong KW, Rosa V, Thuyen T, Seneviratne CJ. Streptococcus mutans forms xylitol-resistant biofilm on excess adhesive flash in novel ex-vivo orthodontic bracket model. Am J Orthod Dentofacial Orthop. 2017;151(4):669-77. doi: 10.1016/j. ajodo.2016.09.017 
7- Richter $A E$, Arruda $A O$, Peters $M C$, Sohn W. Incidence of caries lesions among patients treated with comprehensive orthodontics. Am J Orthod Dentofacial Orthop. 2011;139(5):657-64. doi: $10.1016 / \mathrm{j}$ ajodo.2009.06.037

8- Altmann AS, Collares FM, Ogliari FA, Samuel SM. Effect of methacrylated-based antibacterial monomer on orthodontic adhesive system properties. Am J Orthod Dentofacial Orthop. 2015;147(4 Suppl):S82-7. doi: 10.1016/j.ajodo.2015.01.015

9- Uysal T, Amasyali M, Ozcan S, Koyuturk AE, Sagdic D. Effect of antibacterial monomer-containing adhesive on enamel demineralization around orthodontic brackets: an in-vivo study. Am J Orthod Dentofacial Orthop. 2011;139(5):650-6. doi: 10.1016/j.ajodo.2009.06.038 10- Degrazia FW, Leitune VC, Garcia IM, Arthur RA, Samuel SM, Collares FM. Effect of silver nanoparticles on the physicochemical and antimicrobial properties of an orthodontic adhesive. J Appl Oral Sci. 2016;24(4):404-10. doi: 10.3390/nano10081466

11- Melo MA, Wu J, Weir MD, Xu HH. Novel antibacterial orthodontic cement containing quaternary ammonium monomer dimethylaminododecyl methacrylate. J Dent. 2014;42(9):1193-201. doi: $10.1016 /$ j.jdent.2014.07.006

12- Nascimento $P$, Meereis CT, Maske TT, Ogliari FA, Cenci MS, Pfeifer CS, et al. Addition of ammonium-based methacrylates to an experimental dental adhesive for bonding metal brackets: carious lesion development and bond strength after cariogenic challenge. Am J Orthod Dentofacial Orthop. 2017;151(5):949-56. doi: 10.1016/j. ajodo.2016.10.028

13- Zhou H, Weir MD, Antonucci JM, Schumacher GE, Zhou XD, Xu $\mathrm{HH}$. Evaluation of three-dimensional biofilms on antibacterial bonding agents containing novel quaternary ammonium methacrylates. Int J Oral Sci. 2014;6(2):77-86. doi: 10.1038/ijos.2014.18

14- Cheng L, Weir MD, Xu HH, Antonucci JM, Kraigsley AM, Lin NJ, et al. Antibacterial amorphous calcium phosphate nanocomposites with a quaternary ammonium dimethacrylate and silver nanoparticles. Dent Mater. 2012;28(5):561-72. doi: 10.1016/j.dental.2012.01.005 15- Li F, Weir MD, Chen J, Xu HH. Comparison of quaternary ammoniumcontaining with nano-silver-containing adhesive in antibacterial properties and cytotoxicity. Dent Mater. 2013;29(4):450-61. doi: 10.1016/j.dental.2013.01.012

16- Li F, Weir MD, Chen J, Xu HH. Effect of charge density of bonding agent containing a new quaternary ammonium methacrylate on antibacterial and bonding properties. Dent Mater. 2014;30(4):433-41. doi: 10.1016/j.dental.2014.01.002

17- Li F, Weir MD, Xu HH. Effects of quaternary ammonium chain length on antibacterial bonding agents. J Dent Res. 2013;92(10):932-8. doi: $10.1177 / 0022034513502053$

18- Rego GF, Vidal ML, Viana GM, Cabral LM, Schneider LFJ, Portela MB, et al. Antibiofilm properties of model composites containing quaternary ammonium methacrylates after surface texture modification. Dent Mater. 2017;33(10):1149-56. doi: 10.1016/j.dental.2017.07.010 19- Vidal ML, Rego GF, Viana GM, Cabral LM, Souza JP, Silikas N, et al. Physical and chemical properties of model composites containing quaternary ammonium methacrylates. Dent Mater. 2018;34(1):14351. doi: 10.1016/j.dental.2017.09.020

20- Antonucci JM, Zeiger DN, Tang K, Lin-Gibson S, Fowler BO, Lin NJ. Synthesis and characterization of dimethacrylates containing quaternary ammonium functionalities for dental applications. Dent Mater. 2012;28(2):219-28. doi: 10.1016/j.dental.2011.10.004
21- Park J, Ye Q, Topp EM, Misra A, Kieweg SL, Spencer P. Effect of photoinitiator system and water content on dynamic mechanical properties of a light-cured bisGMA/HEMA dental resin. J Biomed Mater Res A. 2010;93(4):1245-51. doi: 10.1002/jbm.a.32617

22- Wang $Y$, Spencer $P$, Yao X, Ye Q. Effect of coinitiator and water on the photoreactivity and photopolymerization of HEMA/camphoquinonebased reactant mixtures. J Biomed Mater Res A. 2006;78(4):721-8. doi: 10.1002/jbm.a.30733

23- Bertolini MM, Portela MB, Curvelo JA, Soares RM, Lourenco EJ, Telles DM. Resins-based denture soft lining materials modified by chlorhexidine salt incorporation: an in vitro analysis of antifungal activity, drug release and hardness. Dent Mater. 2014;30(8):793-8. doi: 10.1016/j.dental.2014.05.004

24- Martini Garcia I, Jung Ferreira C, Souza VS, Castelo Branco Leitune V, Samuel SM, Souza Balbinot G, et al. Ionic liquid as antibacterial agent for an experimental orthodontic adhesive. Dent Mater. 2019;35(8):1155-65. doi: 10.1016/j.dental.2019.05.010

25- Feng $X$, Zhang $N$, Xu HH, Weir MD, Melo MAS, Bai $Y$, et al. Novel orthodontic cement containing dimethylaminohexadecyl methacrylate with strong antibacterial capability. Dent Mater. 2017;36(5):669-76. doi: $10.4012 / \mathrm{dmj} .2016-370$

26- Shanmugam K, Sarveswari HB, Udayashankar A, Swamy SS, Pudipeddi A, Shanmugam T, et al. Guardian genes ensuring subsistence of oral Streptococcus mutans. Critical reviews in microbiology. 2020;46(4):475-91. doi: 10.1080/1040841X.2020.1796579

27- Ferracane JL, Greener EH. The effect of resin formulation on the degree of conversion and mechanical properties of dental restorative resins. J Biomed Mater Res. 1986;20(1):121-31. doi: 10.1002/ jbm. 820200111

28- Ghadirian H, Geramy A, Najafi F, Heidari S, Golshahi H. Effect of quaternary ammonium salt on shear bond strength of orthodontic brackets to enamel. J Dent (Tehran). 2017;14(3):159-64.

29- Van Meerbeek B, Peumans M, Poitevin A, Mine A, Van Ende $A$, Neves $A$, et al. Relationship between bond-strength tests and clinical outcomes. Dent Mater. 2010;26(2):e100-21. doi: 10.1016/j. dental.2009.11.148

30- Cheng L, Weir MD, Zhang K, Arola DD, Zhou X, Xu HH. Dental primer and adhesive containing a new antibacterial quaternary ammonium monomer dimethylaminododecyl methacrylate. J Dent. 2013;41(4):345-55. doi: 10.1016/j.jdent.2013.01.004

31- Yu F, Dong Y, Yu HH, Lin PT, Zhang L, Sun X, et al. Antibacterial activity and bonding ability of an orthodontic adhesive containing the antibacterial monomer 2-methacryloxylethyl hexadecyl methyl ammonium bromide. Sci Rep. 2017;7:41787. doi: 10.1038/srep41787 32- Pallikari F, Iosifidou S. A study of aging in dental composite using IR and Raman spectroscopy. e-Polymers. 2013;4(1):1-10. doi: 10.1515/ epoly.2004.4.1.793

33- Gaglianone LA, Lima AF, Goncalves LS, Cavalcanti AN, Aguiar FH, Marchi GM. Mechanical properties and degree of conversion of etchand-rinse and self-etch adhesive systems cured by a quartz tungsten halogen lamp and a light-emitting diode. J Mech Behav Biomed Mater. 2012;12:139-43. doi: 10.1016/j.jmbbm.2012.01.018

34- Collares FM, Ogliari FA, Zanchi CH, Petzhold CL, Piva E, Samuel SM. Influence of 2-hydroxyethyl methacrylate concentration on polymer network of adhesive resin. J Adhes Dent. 2011;13(2):125-9. doi: 10.3290/j.jad.a18781 